02;04;09;15

\title{
CuBr-лазер с высоким КПД в режиме сдвоенных импульсов накачки
}

\author{
(C) А.И. Федоров ${ }^{1}$, Д.В. Шиянов ${ }^{1,2}$ \\ ${ }^{1}$ Институт оптики атмоссреры им. В.Е. Зуева СО РАН, Томск \\ ${ }^{2}$ Национальный исследовательский Томский политехнический \\ университет \\ E-mail: fedorov@asd.iao.ru
}

Поступило в Редакцию 27 сентября 2016 г.

Приведены результаты исследований характеристик $\mathrm{CuBr}$-лазера, работающего в режиме сдвоенных импульсов накачки с частотой повторения импульсов генерации $50 \mathrm{~Hz}$. Рассмотрены возможности повышения эффективности лазера за счет выбора оптимальных напряжений диссоциирующего и возбуждающего импульсов и их временной задержки. Показано, что за счет оптимизации и согласованного ввода энергии импульса возбуждения в плазму активной среды можно КПД лазера увеличить более чем в 2 раза. Получен максимальный КПД лазера $2.6 \%$ по импульсу возбуждения со средней мощностью $16 \mathrm{~mW}$, энергией $0.32 \mathrm{~mJ}$, длительностью импульса излучения на полувысоте $40 \mathrm{~ns}$ и временной задержкой $150 \mu \mathrm{s}$. Максимальная средняя мощность излучения равнялась $37 \mathrm{~mW}$ с энергией $0.7 \mathrm{~mJ}$ и КПД лазера $0.7 \%$ для удельной энергии диссоциирующего $24 \mathrm{~mJ} / \mathrm{cm}^{3}$ и возбуждающего $2.7 \mathrm{~mJ} / \mathrm{cm}^{3}$ импульсов накачки.

DOI: 10.21883/PJTF.2017.05.44356.16501

В лазерах на парах галогенидов металлов (ЛПГМ) удается реализовывать более высокие параметры генерации [1], чем в лазерах на парах чистых металлов [2,3]. Основные исследования проводятся в лазерах на парах меди или бромиде меди [1]. В основном ЛПГМ работают в режиме регулярных импульсов накачки с частотой повторения порядка десятков килогерц, в которых энергия разряда используется как для создания активной среды, так и для ее возбуждения. При этом импульсная энергия излучения оказывается низкой, что ограничивает использование этого режима в задачах технологического плана, где требуются высокие энергии излучения с частотой повторения десятки и сотни герц. Данный режим генерации возможен при возбуждении 
активной среды CuBr-лазера сдвоенными импульсами накачки при наличии внешнего нагревателя газоразрядной трубки [4].

Впервые метод сдвоенных импульсов накачки был применен авторами работы [5] для активной среды хлорида меди с буферным газом гелием, в которой при частоте повторения импульсов возбуждения $5 \mathrm{~Hz}$ была получена удельная энергия излучения $17 \mu \mathrm{J} / \mathrm{cm}^{3}$. Первый импульс накачки коммутировался тиратроном и использовался для диссоциации молекул $\mathrm{CuCl}$, второй импульс накачки формировался разрядником с временной задержкой между импульсами от 120 до $200 \mu$ s для возбуждения паров атомов меди. В работе [6] авторами впервые была получена генерация в парах $\mathrm{CuBr}$ при частоте повторения $10 \mathrm{~Hz}$ и оптимальной временной задержке между импульсами накачки от 20 до $100 \mu \mathrm{s}$, которая зависела от давления буферного газа гелия. Были получены импульсы генерация длительностью на полувысоте $20 \mathrm{~ns}$ с энергией $0.4 \mathrm{~mJ}$ и КПД, равным $0.3 \%$ по импульсу возбуждения. Нами в работе [4] были проведены исследования условий получения и возбуждения паров $\mathrm{CuBr}$ при частоте повторения $50 \mathrm{~Hz}$. Была показана возможность повышения эффективности лазера в несколько раз за счет оптимизации параметров импульса возбуждения и его согласования с импедансом плазмы активной среды, образуемой диссоциирующим импульсом накачки. Был получен максимальный КПД лазера $1.2 \%$ по импульсу возбуждения с энергией $0.2 \mathrm{~mJ}$, средней мощностью $10 \mathrm{~mW}$ и длительностью импульса излучения на полувысоте $30 \mathrm{~ns}$ для временной задержки $50 \mu \mathrm{s}$. Таким образом, оставался открытым вопрос о возможности дальнейшего повышения КПД лазера за счет оптимизации параметров диссоциирующего и возбуждающего импульсов накачки и их временной задержки.

В работе рассмотрена возможность повышения энергетических характеристик и КПД лазера за счет оптимизации параметров импульсов накачки в режиме согласованного ввода энергии возбуждения в плазму активной среды. Исследования проводились на установке, описанной в работе [7], которая состояла из газоразрядной трубки (ГРТ), двух импульсных генераторов накачки, генератора временных задержек и системы регистрации параметров разряда и генерации излучения. Конструкция ГРТ приведена в работе [8]. Лазерная трубка имела внутренний диаметр $1 \mathrm{~cm}$ и активную длину $40 \mathrm{~cm}$ с рабочим объемом $31.4 \mathrm{~cm}^{3}$, которая нагревалась от внешней печи. На холодных концах трубки были расположены внутренние электроды и выходные окна. Резонатор состо-

Письма в ЖТФ, 2017, том 43, вып. 5 
ял из Al-зеркала и кварцевой пластины. К электродам прикладывались два импульса накачки, временная задержка $\left(\tau_{d}\right)$ между которыми была оптимальной и равнялась $150 \mu \mathrm{s}$. Первый диссоциирующий импульс накачки обеспечивался высоковольтным источником на $20 \mathrm{kV}$ с рабочей емкостью $\left(C_{d}\right)$, которая могла изменяться от 1.1 до $11.5 \mathrm{nF}$. Он позволял вкладывать в разряд удельную энергию диссоциирующего импульса $\left(E_{d}\right)$ от 2.7 до $24 \mathrm{~mJ} / \mathrm{cm}^{3}$. Для возбуждения атомов меди использовался дополнительный высоковольтный источник питания на $15 \mathrm{kV}$ c рабочей емкостью $\left(C_{p}\right)$, равной $1 \mathrm{nF}$. Он обеспечивал удельную энергию возбуждения $\left(E_{p}\right)$ в активной среде от 0.4 до $3.6 \mathrm{~mJ} / \mathrm{cm}^{3}$ и мощность возбуждения $\left(W_{p}\right)$ от 2 до $17 \mathrm{~kW} / \mathrm{cm}^{3}$. Лазер работал с частотой повторения импульсов возбуждения $50 \mathrm{~Hz}$. В качестве буферного газа использовался неон при давлении 25 Torr. В зависимости от величины рабочего напряжения на плазме и емкости источников диссоциирующего и возбуждающего импульсов накачки можно было управлять вводом удельных энергий в активную среду $\mathrm{CuBr}$-лазера. За счет генератора временных задержек достигалось оптимальное время ввода энергии импульса возбуждения в активную среду, тем самым обеспечивая частичное согласование импедансов источников накачки. Ранее считалось, что увеличение амплитуды импульса возбуждения должно было приводить к увеличению энергии излучения и КПД лазера. Нами было обнаружено, что увеличение удельной энергии или мощности импульса возбуждения влияет на рост энергии или мощности излучения при уменьшении КПД лазера из-за рассогласования импедансов источников накачки [4]. Максимальный КПД лазера соответствовал режиму согласованного ввода энергии возбуждения в плазму активной среды, которая определялась параметрами диссоциирующего импульса накачки. Поэтому нами была проведена оптимизация параметров источников диссоциирующих и возбуждающих импульсов накачки, обеспечивающих как образование плазмы активной среды, так и ее возбуждение.

На рис. 1 приведены зависимости напряжения на плазме и удельной энергии импульса накачки для создания активной среды $\mathrm{CuBr}$-лазера от величины рабочей емкости диссоциирующего источника питания. Оказалось, что он обеспечивал максимальное напряжение на плазме $16 \mathrm{kV}$ для рабочих емкостей от 1 до $4 \mathrm{nF}$. Затем напряжение на плазме линейно спадало до $11 \mathrm{kV}$ с ростом емкости до $11.5 \mathrm{nF}$. При этом удельная энергия накачки линейно росла до $24 \mathrm{~mJ} / \mathrm{cm}^{3}$ при изменении

Письма в ЖТФ, 2017, том 43, вып. 5 


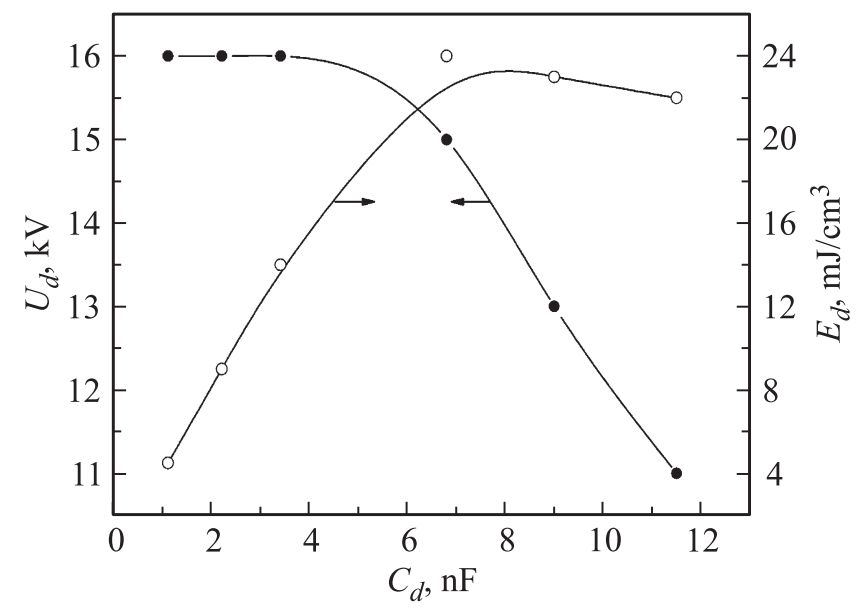

Pис. 1. Зависимости напряжения на плазме и удельной энергии накачки для создания активной среды $\mathrm{CuBr}$-лазера от величины рабочей емкости диссоциирующего источника питания.

емкости от 1 до $6.8 \mathrm{nF}$, а далее медленно спадала до $22 \mathrm{~mJ} / \mathrm{cm}^{3}$ при увеличении емкости до $11.5 \mathrm{nF}$. Нами в работе [7] было показано, что для максимального КПД лазера $1.5 \%$ оптимальной являлась задержка между импульсами накачки $150 \mu \mathrm{s}$. Поэтому эта временная задержка нами использовалась в последующих экспериментах.

На рис. 2 приведены зависимости средней мощности излучения и КПД лазера от величины рабочей емкости источника диссоциирующего импульса накачки и напряжения на плазме активной среды для оптимальной емкости $1 \mathrm{nF}$ источника импульса возбуждения. Средняя мощность излучения резко возрастала для максимального напряжения возбуждения $15 \mathrm{kV}$ с увеличением диссоциирующей емкости до $6.8 \mathrm{nF}$ и соответствовала $37 \mathrm{~mW}$, а затем она медленно уменьшалась до $35 \mathrm{~mW}$ при $C_{d}=11.5 \mathrm{nF}$. Для КПД лазера наблюдалась иная картина. Это его медленное нарастание до максимального значения $0.7 \%$ с увеличением диссоциирующей емкости до $6.8 \mathrm{nF}$, а затем его медленный спад. Согласованный режим ввода энергии возбуждения предложен в [4]. Этот режим нами наблюдался при более низких напряжениях на плазме активной среды. Оказалось, что оптимальным напряжением

Письма в ЖТФ, 2017, том 43, вып. 5 


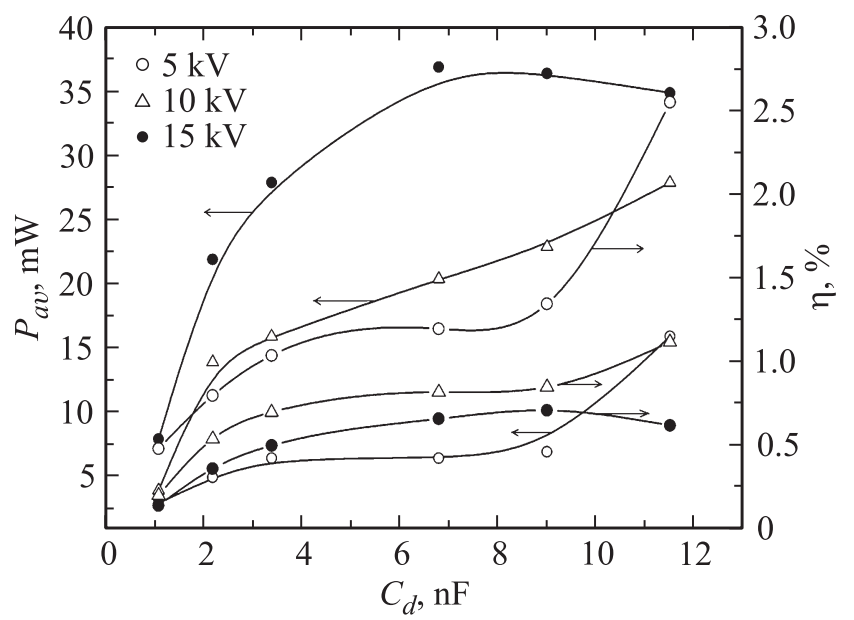

Рис. 2. Зависимости средней мощности излучения и КПД лазера от величины рабочей емкости диссоциирующего источника питания и напряжения импульса возбуждения на плазме активной среды для $C_{p}=1 \mathrm{nF}$ и $\tau_{d}=150 \mu \mathrm{s}$.

на плазме является величина $5 \mathrm{kV}$. Зависимости средней мощности излучения и КПД лазера в случае согласованного режима отличались от этих зависимостей для режима максимального напряжения на плазме $15 \mathrm{kV}$. Средняя мощность излучения нарастала с ростом диссоциирующий емкости с 1.1 до $3.3 \mathrm{nF}$, а затем оставалась почти постоянной и равнялась $6.5 \mathrm{~mW}$ при увеличении емкости до $9 \mathrm{nF}$. Она резко возрастала до $16 \mathrm{~mW}$ с ростом емкости, которая увеличивалась до $11.5 \mathrm{nF}$. Аналогичная картина наблюдалась для КПД лазера, который был также максимальным для емкости $11.5 \mathrm{nF}$ и равнялся 2.6\%. Этот факт говорит о том, что оптимальные условия образования плазмы активной среды наблюдались при емкости $11.5 \mathrm{nF}$ с удельной энергией диссоциирующего импульса накачки, равного $22 \mathrm{~mJ} / \mathrm{cm}^{3}$. При напряжении возбуждения $10 \mathrm{kV}$ наблюдались наиболее оптимальные параметры излучения. Это средняя мощность излучения $28 \mathrm{~mW}$, энергия излучения $0.56 \mathrm{~mJ}$ с КПД лазера $1.1 \%$. Таким образом, за счет оптимального напряжения согласованного режима возбуждения $5 \mathrm{kV}, E_{p}=0.4 \mathrm{~mJ} / \mathrm{cm}^{3}$ и $W_{p}=2 \mathrm{~kW} / \mathrm{cm}^{3}$ нами был получен максимальный КПД лазера $2.6 \%$

Письма в ЖТФ, 2017, том 43, вып. 5 


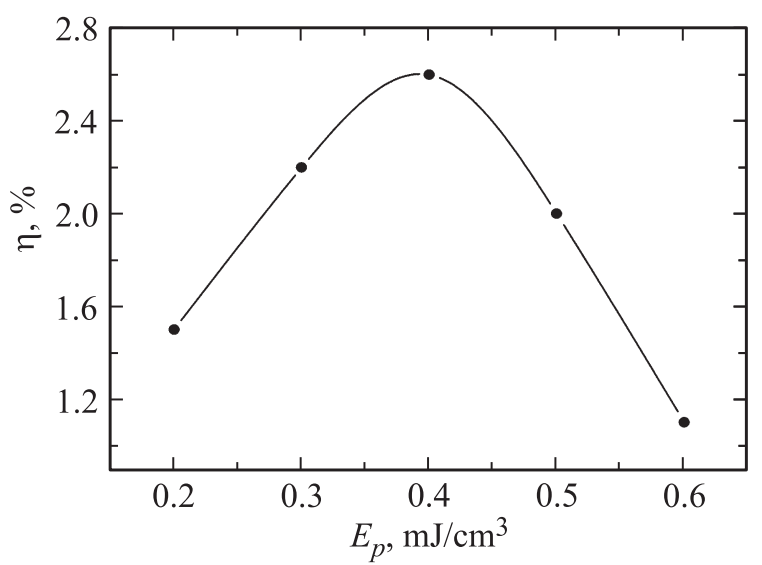

Рис. 3. Зависимость КПД лазера от удельной энергии импульса возбуждения в режиме согласованного ввода ее в плазму активной среды для $C_{d}=11.5 \mathrm{nF}$, $C_{p}=1 \mathrm{nF}$ и $\tau_{d}=150 \mu \mathrm{s}$.

со средней мощностью излучения $16 \mathrm{~mW}$, энергией излучения $0.32 \mathrm{~mJ}$ при длительности импульса генерации на полувысоте $40 \mathrm{~ns}$. Для максимального напряжения возбуждения на плазме $15 \mathrm{kV}, E_{p}=2.7 \mathrm{~mJ} / \mathrm{cm}^{3}$ и $W_{p}=17 \mathrm{~kW} / \mathrm{cm}^{3}$ средняя мощность излучения равнялась $37 \mathrm{~mW}$, энергия излучения $0.7 \mathrm{~mJ}$ и КПД лазера $0.7 \%$ при длительности импульса генерации $40 \mathrm{~ns}$. Для напряжения возбуждения $10 \mathrm{kV}, E_{p}=1.6 \mathrm{~mJ} / \mathrm{cm}^{3}$ и $W_{p}=8 \mathrm{~kW} / \mathrm{cm}^{3}$ средняя мощность $28 \mathrm{~mW}$, энергия излучения $0.56 \mathrm{~mJ}$ и КПД лазера $1.1 \%$ при длительности импульса генерации $30 \mathrm{~ns}$. Нами было обнаружено, что максимальный КПД лазера наблюдался при минимальных энергиях импульса возбуждения.

На рис. 3 приведена оптимальная зависимость КПД лазера от удельной энергии возбуждения в режиме согласованного ввода ее в плазму активной среды. Для минимальной энергии возбуждения $0.2 \mathrm{~mJ} / \mathrm{cm}^{3}$ и напряжении на плазме $3 \mathrm{kV}$ КПД равнялся $1.5 \%$, а для максимальной энергии возбуждения $0.6 \mathrm{~mJ} / \mathrm{cm}^{3}$ и напряжении $6 \mathrm{kV}$ он равнялся $1.1 \%$. Максимальный КПД лазера $2.6 \%$ наблюдался при оптимальной удельной энергии возбуждения $0.4 \mathrm{~mJ} / \mathrm{cm}^{3}$.

Таким образом, нами показано, что для повышения КПД лазера необходим режим, который обеспечивал бы оптимальный ввод энергии

Письма в ЖТФ, 2017, том 43, вып. 5 
импульса возбуждения в плазму активной среды. Он достигался согласованием импедансов источников накачки с плазмой и осуществлялся за счет выбора оптимальных напряжений диссоциирующего и возбуждающего импульсов и их временной задержки. Получен максимальный КПД лазера $2.6 \%$ для импульса возбуждения с энергией излучения $0.32 \mathrm{~mJ}$, средней мощностью излучения $16 \mathrm{~mW}$ и длительностью импульса генерации $40 \mathrm{~ns}$ для временной задержки $150 \mu \mathrm{s}$, которому соответствовала удельная энергия диссоциирующего импульса накачки $22 \mathrm{~mJ} / \mathrm{cm}^{3}$ и возбуждающего импульса $-0.4 \mathrm{~mJ} / \mathrm{cm}^{3}$.

\section{Список литературы}

[1] Батенин В.М., Бучанов В.В., Казарян М.А. и др. Лазеры на самоограниченных переходах атомов металлов. М.: Научная книга, 1998.

[2] Земсков К.И., Исаев А.А., Петраш Г.Г. // Квант. электроника. 1997. Т. 24. № 7. С. 596.

[3] Петраш Г.Г. // Изв. вузов. Физика. 1999. № 8. С. 18.

[4] Федоров А.И., Шиянов Д.В. // Письма в ЖТФ. 2015. Т. 41. В. 15. С. 98.

[5] Chen C.J., Nerheim N.M., Russell G.R. // Appl. Phys. Lett. 1973. V. 23. N 29. P. 514.

[6] Абросимов Г.В., Васильцов В.В., Волочин В.Н. и др. // Письма в ЖТФ. 1976. T. 2. B. 9. C. 417.

[7] Федоров А.И., Шиянов Д.В. // Оптика атмосферы и океана. 2015. Т. 28. № 11. C. 1035.

[8] Федоров А.И., Федоров В.Ф., Димаки В.А. // Оптика атмосферы и океана. 2012. T. 25. № 2. C. 198. 